\title{
El uso, apropiación e impacto de las TIC por las mujeres rurales jóvenes en el Perú
}

\author{
Andrea García Abad, Mariana Barreto Ávila
}

\begin{abstract}
Resumen
El presente estudio surge a partir de los hallazgos obtenidos por el equipo de Perú del programa Nuevas Trenzas, esfuerzo de investigación desarrollado en seis países de América Latina, bajo la coordinación del Instituto de Estudios Peruanos. El programa busca profundizar el conocimiento sobre los cambios recientes en las vidas las mujeres rurales jóvenes. Este estudio se centrará en los actores, las mujeres rurales jóvenes, para analizar los usos y las formas de apropiación de las TIC, específicamente teléfono móvil e Internet, para averiguar qué impacto tienen estos procesos en sus dinámicas cotidianas y estrategias de vida. Asimismo, buscaremos discutir el efecto de las nuevas prácticas y experiencias que surgen a partir del uso de estas tecnologías tienen algún efecto en los márgenes de autonomía de este colectivo. El estudio presentará los hallazgos obtenidos durante el trabajo de campo en dos centros poblados rurales del Perú, donde se realizaron grupos focales y entrevistas en profundidad con mujeres rurales jóvenes. Si bien es un trabajo exploratorio, nuestra intención es contribuir a la discusión sobre los retos de las TIC para convertirse en herramientas que mitiguen las brechas de desigualdad que afectan a las mujeres rurales jóvenes, colectivo clave para el desarrollo rural.
\end{abstract}

Palabras claves: mujeres rurales jóvenes, telefonía móvil, Internet, Perú 


\section{Introducción}

Nuevas Trenzas es un esfuerzo de investigación desarrollado en seis países de América Latina, bajo la coordinación del Instituto de Estudios Peruanos, con el apoyo financiero de la División de América Latina y el Caribe del Fondo Internacional para el Desarrollo Agrícola (FIDA)'. El objetivo es profundizar en nuestro conocimiento sobre las nuevas generaciones de mujeres rurales jóvenes de América Latina, sus retos, perspectivas, capacidades y percepciones, con el fin de aportar en el diseño de políticas y programas de desarrollo rural más eficientes y con mayores posibilidades de conjugar los objetivos de reducción de la pobreza e inclusión social².

El trabajo de campo realizado como parte de la primera etapa del programa partía de un enfoque bastante amplio, en tanto buscaba delinear el perfil de las mujeres rurales jóvenes en cada uno de estos países, tomando en cuenta diferentes aspectos de sus vidas, educación, experiencias laborales, relaciones familiares, entre otros. En el caso de Perú, uno de los aspectos contemplados fueron las tecnologías de la información y de la comunicación (TIC), que resultó ser uno de los temas más recurrentes durante los grupos focales y entrevistas con las jóvenes.

La información cualitativa que analizaremos en este documento fue recolectada durante las visitas que realizamos a los centros poblados de Nuevo Pedregal, en la costa norte, y Andaray, en la sierra sur del país, entre los meses de febrero y abril de 2012. En estas localidades realizamos grupos focales segmentados por edades y entrevistas a profundidad con mujeres jóvenes entre 14 y 35 años. Se llevó a cabo, también, un grupo focal en cada localidad con mujeres adultas mayores de 35 años, para conocer su percepción sobre la telefonía móvil e Internet y sobre el uso que hacen las jóvenes de esas tecnologías.

Este documento pretende ser una primera aproximación a las relaciones entre las TIC y las mujeres rurales jóvenes, colectivo que consideramos clave para el desarrollo rural. Buscamos discutir si las nuevas prácticas y experiencias que surgen a partir del uso de las TIC tienen algún efecto en los márgenes de autonomía de las mujeres rurales y en su relación con padres, madres y pares masculinos. A la luz de ello, planteamos las siguientes preguntas que guiarán nuestro análisis:

- ¿De qué manera el colectivo de mujeres rurales jóvenes se apropia de los teléfonos móviles e Internet?

- ¿Qué impacto tienen el acceso y uso de estas tecnologías en las dinámicas sociales cotidianas de las mujeres rurales jóvenes?

\footnotetext{
1 Los paises incluidos en Nuevas Trenzas son Colombia, Ecuador, El Salvador, Guatemala, Nicaragua y Perú. Para más información sobre el programa: www.nuevastrenzas.org

2 Nuevas Trenzas considera "mujeres jóvenes" el rango de edad entre 14 y 35 años. Este grupo se divide en tres segmentos: mujeres adolescentes (14-17 años), mujeres en etapa de transición (18-25 años) y adultos jóvenes (26-35 años).
} 
- ¿Cuál es el alcance de las TIC en los procesos de autonomía de las mujeres rurales jóvenes?

Esperamos que el documento contribuya a la discusión sobre la necesidad de diseñar políticas relacionadas con el acceso a las TIC que otorguen más importancia a los usuarios y que satisfagan sus necesidades de información y comunicación, de manera tal que se impulse su potencial como herramientas para incrementar las libertades y capacidades, en favor de un desarrollo individual más autónomo y de un proceso de desarrollo rural más integral y equitativo ${ }^{3}$.

\section{TIC y el desarrollo rural}

El incremento de iniciativas estatales y proyectos dedicados a ampliar una infraestructura que permita una mayor penetración de la telefonía móvil y conectividad a Internet en las zonas rurales, es un fenómeno que se viene dando en toda la región latinoamericana (Bustamante et al 2009). Este esfuerzo sostenido por extender el acceso a las TIC refleja una visión generalizada en torno a estas tecnologías que se conciben como vía para mitigar la exclusión social y el desarrollo desigual entre las zonas rurales. Sin embargo, muchos de estos esfuerzos han ignorado que el acceso físico a estas nuevas tecnologías no garantiza un acceso igualitario de los diferentes sectores de la población rural (Donner 2008, Calcina 2013). Asimismo, estos esfuerzos no siempre toman en cuenta que el proceso de adaptación o manipulación por parte de los usuarios son muy distintos, según sus características y sus contextos particulares. Con el término "apropiación" nos referimos al "proceso material y simbólico de interpretación y dotación de sentido respecto de un determinado artefacto cultural por parte de un grupo social, enfatizando la capacidad de los sujetos para volverlo significativo de acuerdo con sus propósitos" (Benitez Larghi et al 2012: 33). El desarrollo que las nuevas TIC dependerá del uso que los individuos hagan de ellas, así como de las políticas de acceso. Las TIC pueden convertirse en herramientas que mejoren la calidad de vida y contribuyan al bienestar de las personas, pero también pueden jugar un rol conservador y reaccionario (Buskens y Webb 2009: 4), que refuerce las relaciones de poder existentes, tanto a nivel político y económico, como étnico y de género. Burrel señala que la irrupción de nuevas tecnología, y en concreto de Internet, puede propiciar una inflación de expectativas en la población local y, al mismo tiempo una disrupción de la cotidianidad, que obliga a mecanismos de ajuste, que van desde rumores hasta nuevas prácticas de sociabilidad (Burrell 2010).

3 Una versión previa de esta investigación se presentó en el Seminario Internacional sobre Escalamiento de Innovaciones Rurales, organizado en Lima por el instituto de Estudios Peruanos y el International Deveolpment Research Centre en mayo de 2012 (Barreto y Garcia 2013). 
Para lograr la inclusión digital se necesita más que la expansión física de las tecnologías. Es necesario tomar en cuenta los procesos locales, demandas, necesidades y capacidades de los usuarios (Hopkins et al 2013). Es importante considerar el acceso y uso efectivo, es decir, la capacidad para adaptar, manipular y generar conocimiento para el beneficio concreto de un individuo u organización (Bustamante et al 2009: 3). En este sentido, por lo general, el impacto de Internet y de la telefonía móvil ha sido estudiado desde su relación con la educación o con el ámbito laboral y productivo. No obstante, existen otros importantes efectos en la vida social y afectiva (Aronés et al 2011: 81). Las TIC contribuyen a redefinir las interacciones y las relaciones familiares, amicales y de pareja. La privacidad, la interacción y la formación de relaciones sociales son procesos muy importantes, principalmente durante la etapa de la juventud. Como argumenta León (2010), la comunicación entre pares y las relaciones interpersonales constituyen durante esta etapa una parte importante de la construcción de la propia identidad. Internet y la telefonía móvil ocupan un espacio nuevo de encuentro, de conversación, de comunicación.

\section{TIC, mujeres y autonomía}

La importancia de estudiar la apropiación de las TIC, más allá de su acceso y uso, recae en el hecho de que el valor que las tecnologías tienen para los usuarios dependerá de los, muchas veces múltiples, significados que ellos les otorguen, que al mismo tiempo, dependerá de diversos factores como el contexto, el género, la pertenencia generacional y la biografía personal.

De acuerdo con el Censo Nacional de 2007, en Perú viven 27 millones de personas. De ellas, el 24 por ciento vive en zonas rurales, el 49 por ciento son hombres y el 51 por ciento mujeres. Un poco más de 34 por ciento de la población total del país es joven de acuerdo con la definición adoptada por Nuevas Trenzas. Las mujeres rurales jóvenes son el total 1.301.760 personas, que representan el cuatro por ciento de la población total nacional, el dieciséis por ciento respecto a la población rural, el ocho por ciento respecto al total de mujeres del país y el doce por ciento respecto a la población joven nacional.

En la actualidad, uno de cada nueve jóvenes peruanos es una mujer rural. Se trata de un colectivo que está inmerso en un proceso de cambio muy fuerte (Agüero y Barreto 2012, Ames 2013). Las jóvenes rurales tienen hoy expectativas educativas, laborales y de vida distintas a las que tenían las generaciones anteriores. También, sus pautas de consumo, demandas y patrones de movilidad han cambiado. La juventud rural de hoy tiene nuevas-y quizás mayores-posibilidades así como un gran potencial para insertarse en las dinámicas territoriales y aportar desde su posición al desarrollo territorial, aunque también enfrenta importantes retos y limitaciones (Barreto y García 2012). 
Todas estas cuestiones se reflejan en el uso de las TIC. La estructura jerárquica de las sociedades rurales genera patrones culturales que ponen a las mujeres en una situación de desventaja para desenvolverse en la vida cotidiana, para movilizarse, entrar al mercado laboral e, incluso, para acceder y hacer uso de las TIC. Estas normas sociales con sesgos de género limitan en gran medida la autonomía de las mujeres, es decir, su capacidad para acceder al control de recursos sociales y materiales y de tomar decisiones en el ámbito personal, familiar y público (Banco Mundial 2012).

Si bien la expansión del acceso físico de las TIC en el territorio rural pone esta tecnología aparentemente al alcance de la mayoría de los habitantes, existen factores sociales que condicionan su apropiación desigual. Diversos estudios muestran que el uso del teléfono móvil e Internet se diferencia por género (Archambault 2011, León 2013). Sin embargo, también es cierto que existe una tendencia general, de la que participan mujeres y hombres a que cada vez tengan un mayor protagonismo en sus vidas. Como afirman Hahn y Kibora (2008: 88), las TIC son una manera de "estar al día" con las tendencias globales. Se han convertido en íconos de la comunicación sin fronteras, que especialmente en los países en desarrollo proyectan una imagen de modernidad, autonomía y cosmopolitismo.

La apropiación de estas tecnologías por parte de las jóvenes rurales puede abrir nuevos espacios de interacción, negociación y también de reflexión que, a su vez, pueden resultar en el desarrollo de estrategias de vida más autónomas. Una mayor autonomía permitiría a las mujeres pasar de la conformidad ante las normas de género desiguales a su cuestionamiento, mediante nuevas aspiraciones, y la habilidad de buscar y lograr las metas esperadas (Banco Mundial 2012). De esta manera, la autonomía de las mujeres es una condición necesaria para mitigar las brechas de desigualdad que las afectan. En las siguientes páginas analizamos hasta qué punto las nuevas tecnologías aportan en este camino.

\section{Metodología: dos estudios de caso}

La información trabajada como parte del programa Nuevas Trenzas, nos brinda una primera entrada a la relación entre TIC, mujeres rurales jóvenes y brechas de desigualdad. Un primer dato es que, con relación a la tenencia de teléfonos celulares en los hogares, existe una marcada brecha entre las mujeres rurales jóvenes y sus contrapartes urbanas: 89 por ciento de las mujeres jóvenes urbanas tiene al menos un celular en su hogar, frente a 52 por ciento de sus pares rurales. Esta brecha crece conforme aumenta la edad. Un segundo dato a considerar es que, sin embargo, dentro del grupo de mujeres rurales jóvenes, existen diferencias importantes, dependiendo del nivel de pobreza de los hogares. Aproximadamente el 80 por ciento de mujeres rurales jóvenes que viven en hogares no pobres cuenta al menos con un celular en el hogar, porcentaje que se reduce considerablemente mientras mayor es la pobreza (Agüero y Barreto 2012). 
En cuanto al uso de Internet, encontramos también una marcada brecha geográfica, matizada en este caso sobre todo por cuestiones generacionales, según se observa en el Gráfico 1.

Gráfico 1: Brecha geográfica y uso de Internet.

Porcentaje que accedió al menos una vez a Internet en el último mes según grupos de edad

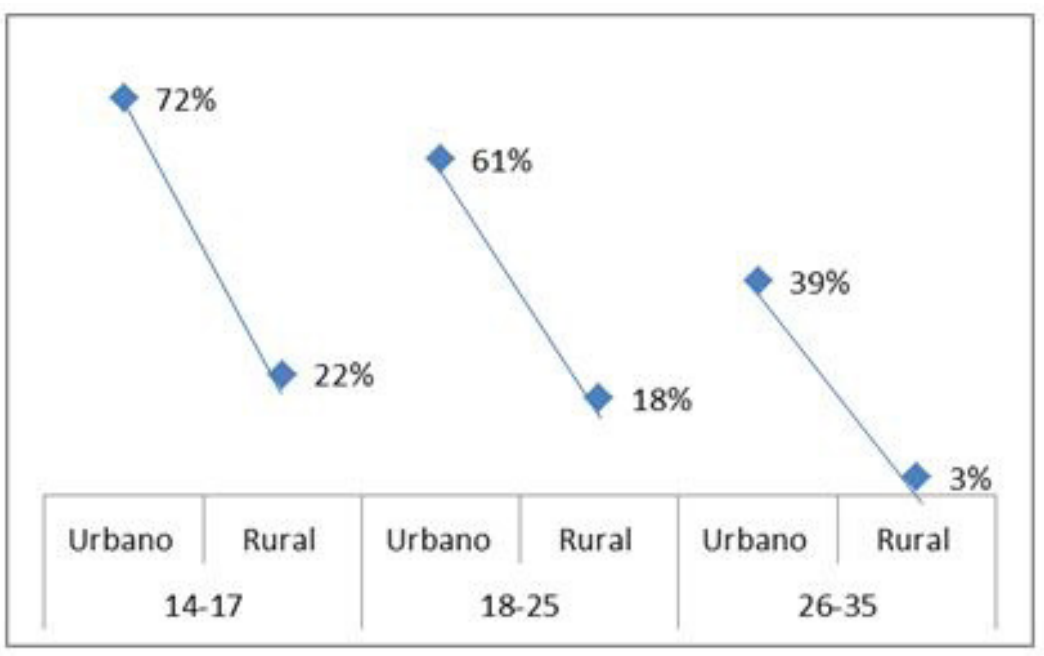

Fuente: ENAHO 2010.

Este gráfico nos muestra dos cosas. Por un lado, evidencia que existe una muy fuerte brecha geográfica en acceso a Internet; por otro lado, muestra también la importancia de la brecha generacional, tanto para las mujeres urbanas como para las rurales. En el caso de este último grupo, un 22 por ciento de las mujeres entre 14 y 17 años accede a Internet, mientras que solo lo hace un tres por ciento de las mayores de 25 años. En el caso de la mujeres rurales también es importante la brecha de pobreza, aunque las diferencias son más pequeñas en los grupos de menor edad: las jóvenes no pobres entre 14 y 17 años usan casi tres veces más Internet que sus pares pobres, y seis veces más que las pobres extremo. En el siguiente grupo de edad, hay una diferencia del doble con respecto a los pobres y multiplica por siete el uso de los pobres extremos.

Para matizar y entender mejor estas cifras, trabajamos en profundidad el uso de TIC por parte de las mujeres rurales jóvenes en dos centros poblados de diferentes regiones de Perú: Nuevo Pedregal, en Piura, y Andaray, en Arequipa. Estas localidades fueron parte del trabajo cualitativo del programa Nuevas Trenzas durante su primera etapa (Agüero y 
Barreto 2012). En ambos casos se realizaron grupos focales y entrevistas en profundidad' A pesar de que ninguna de las herramientas cualitativas fue específicamente construida para analizar en detalle el tema de las TIC, la recurrencia e importancia que las jóvenes le dieron a este tema, nos permitió profundizar en la cuestión.

Nuevo Pedregal es una comunidad principalmente agrícola, ubicada en el distrito de Catacaos, a aproximadamente treinta minutos de la ciudad de Piura, capital del departamento del mismo nombre. Se trata de un centro poblado costeño pobre, pero situado dentro de uno de los territorios considerados más dinámicos del país, lo que como veremos tiene efectos paradójicos sobre las mujeres rurales jóvenes. Su historia es resultado de varias olas de invasiones de terrenos que comenzaron hace aproximadamente quince años y en la actualidad tiene alrededor de mil habitantes. Muchos de los hogares de Nuevo Pedregal carecen de servicios básicos. Tampoco hay escuela primaria o secundaria o posta de salud, y solo hay un centro de educación inicial. Sin embargo, por estar en una zona no pobre del país, la población no es beneficiaria de muchos programas sociales del Estado y tampoco cuenta con intervenciones de la cooperación internacional o de ONG locales.

Nuevo Pedregal es un centro poblado en formación, que recibe constantemente nuevos habitantes, generalmente jóvenes de centros poblados vecinos, que buscan construir una vivienda propia. A pesar de la cercanía con la ciudad de Piura, a menos de una hora en bus, las dinámicas de movilización no son tan frecuentes como esperábamos y en general, los habitantes de Nuevo Pedregal no tienen vínculos estrechos o dinámicas cotidianas con la capital del departamento. A pesar de que todas las empresas de telefonía móvil tienen alcance en Nuevo Pedregal, son pocos los hogares que cuentan con un aparato. En la mayoría de casos, el celular es compartido por los miembros de la familia y permanece en la casa, como si fuera un teléfono fijo. Las personas que no tienen celular se movilizan a Pedregal Grande, el centro poblado vecino, donde está el teléfono público más cercano. El uso de Internet es aún más escaso. No hay servicios de cabinas en el centro poblado, por lo que quienes desean utilizar Internet deben ir a Pedregal Grande, donde funciona una cabina, o a Catacaos, donde funcionan alrededor de seis cabinas.

Andaray es la capital del distrito del mismo nombre, ubicado en la provincia de Condesuyos, en el departamento de Arequipa, a 3.050 metros sobre el nivel del mar. Tiene en la actualidad alrededor de 700 habitantes y se encuentra a aproximadamente ocho horas en autobús de la ciudad de Arequipa. A pesar de esta relativa lejanía, es un caserío bastante dinámico desde el punto de vista social. En Andaray trabaja desde hace algunos años el Proyecto de Desarrollo Sierra Sur. También tiene presencia la Asociación Especializada para el Desarrollo Sostenible (AEDES), una organización no gubernamental que trata temas de derechos humanos y políticos, desarrollo empresarial y democratización de los gobiernos locales.

1 Además, de estas dos localidades se realizaron entrevistas en Yapatera, localidad igualmente situada en el departamento de Piura, caracterizada por su alto porcentaje de población afrodescendiente, el más alto del Perú. 
Hace tres años llegó a Andaray la cobertura de telefonía celular del principal operador privado del país, que hasta el momento de escribir este documento seguía siendo la única empresa que operaba en la zona. Hay dos tipos de servicios que las mujeres entrevistadas contratan cuando van a la ciudad: postpago RPM, con planes mensuales entre 49 a 79 soles, y prepago'. En este último caso las recargas no son muy grandes, alrededor de treinta soles al mes, o a lo mucho diez soles por semana.

Durante nuestra estadía en la localidad no encontramos acceso público a Internet, pero los pobladores afirmaban que esto cambiaría en pocas semanas, gracias a gestiones municipales ${ }^{2}$. El único lugar de la localidad que contaba para ese entonces con acceso era el local de AEDES, donde había un modem portátil que funcionaba con la línea telefónica. Tanto en la municipalidad como en la escuela existían computadoras, pero solamente dos o tres de los pobladores tenían un aparato propio en su casa. Para acceder a Internet los pobladores de Andaray debían ir a Yanaquihua, centro poblado ubicado a cuarenta minutos de distancia a pie, donde existe una cabina pública.

Para analizar la información recogida en estas dos localidades partimos del marco teórico elaborado por Nuevas Trenzas. Como hemos mencionado antes, consideramos que las mujeres rurales jóvenes se encuentran en una situación de desventaja frente a otros grupos de población. Se trata de un colectivo marcado por un conjunto de brechas que se cruzan entre sí, generando desigualdades entrecruzadas, que limitan su capacidad para desarrollar estrategias de vida autónomas e insertarse en los procesos de toma de decisiones. En concreto, para los fines de este estudio nos enfocaremos en tres brechas trabajadas en la primera etapa de Nuevas Trenzas:

- Brecha de lugar de residencia, que separa a las mujeres rurales jóvenes de sus contemporáneas urbanas.

- Brecha de género, que separa a las mujeres rurales jóvenes de los hombres rurales de su mismo grupo de edad.

- Brecha de generación, que separa a las mujeres rurales jóvenes de sus abuelas y madres rurales.

Tomaremos en cuenta, además, otra brecha que consideramos importante en la vida de las mujeres rurales jóvenes, que llamaremos la brecha de convivencia. Esta hace referencia a lo que hemos denominado el "punto de quiebre": momento crítico en las trayectorias personales de las mujeres rurales jóvenes, según las historias de vida levantadas en el curso del programa.

\footnotetext{
1 RPM (Red Privada Movistar) es una opción de comunicación brindada por la empresa Movistar mediante la cual todos aquellos usuarios del servicio pueden comunicarse sin costo entre ellos.

2 En mayo de 2012, en su presentación en el Seminario Internacional sobre Escalamiento de Innovaciones Rurales, organizado en Lima por el IEP y el IDRC, el alcalde de Andaray señaló que el servicio de Internet ya estaba disponible en los locales públicos de la localidad.
} 


\section{Brecha de género: interacción de discursos y prácticas}

Un primer dato a destacar es que en las dos localidades encontramos dinámicas de uso del teléfono celular e Internet diferentes. Estas dinámicas remiten a cuestiones de género, pero no siempre coinciden en Nuevo Pedregal y Andaray. En el caso de Nuevo Pedregal, la localidad más pobre que hemos visitado, los hombres utilizan más el celular y poseen uno con más frecuencia que las mujeres. Para ellos, especialmente para los mayores, el celular es una herramienta de trabajo, a través de la que esperan ser contactados, aunque sea para tareas eventuales. Estos celulares suelen ser de segunda mano, comprados o "heredados" de familiares que pudieron comprarse uno mejor. Suelen ser los modelos más simples, y por lo tanto los más baratos del mercado. Los montos de recarga fluctúan entre los cinco y diez soles, por lo que intentan realizar pocas llamadas para que el saldo les dure alrededor de un mes.

En Andaray, en cambio, las mujeres de los diferentes grupos de edad-incluso las mayores-opinan que son ellas quienes utilizan más el celular que los hombres. Para las mujeres mayores el celular es una importante herramienta de trabajo. Para quienes tienen un negocio propio les permite comunicarse y coordinar con sus clientes o intermediarios, con lo que evitan viajar con demasiada frecuencia a las ciudades cercanas. Además de la utilidad para el trabajo, en todos los grupos de edad, el celular es importante en tanto permite a las mujeres conservar sus relaciones familiares. Esto se da de la misma manera también en Nuevo Pedregal. Ya sea con hijos que estudian o trabajan en la ciudad, con padres o hermanos que viven lejos e incluso con tíos y primos, la posibilidad de poder contactarse con ellos en cualquier momento les brinda tranquilidad, y también, como ellas dicen, las hace sentirse "menos solas".

Las mujeres mayores usan el teléfono celular estrictamente para fines de comunicación, mientras que las jóvenes utilizan sobre todo las funciones "extra": la música y la cámara de fotos. Para este grupo el celular es principalmente un "dispositivo personal": ahí guardan sus mensajes de texto, su música, sus fotos. Por ejemplo, Pamela, una joven de Andaray compró su celular con sus ahorros "para estar en comunicación con sus amigas". Sin embargo, en la práctica lo usa más para escuchar música y tomar fotos.

A la luz de lo recogido en las entrevistas, encontramos, entonces, que el celular satisface, por un lado, la necesidad de comunicación de las jóvenes con personas relacionadas a su trabajo, con sus familiares y con sus amigos. Debemos notar que se trata de una función importante, en la medida en que permite a las mujeres conservar e incluso fortalecer sus redes sociales, lo que en última instancia, aporta a su autonomía. El celular también abre posibilidades de pertenencia a un grupo: las jóvenes se toman fotos que luego comparten, intercambian mensajes, etcétera. Al mismo tiempo, les permite un espacio de privacidad: pueden hablar con sus pares, hombres y mujeres, sin que sus padres las escuchen-por más que ellos digan lo contrario -, pueden intercambiar mensajes 0 fotografías. En última instancia, entonces, el celular más allá de su valor comunicativo: 
tiene valor social, en la medida que permite ganar algo de independencia - o puesto de otra forma, esquiva de cierto modo el control-de los padres o incluso de sus parejas.

En el caso de Internet, las mujeres de ambas localidades afirman que los hombres usan con más frecuencia este medio, aunque esto no quiere decir que ellas no lo usen. Si comparamos entre los grupos de edad, son las mujeres entre 14 y 17 años quienes están más familiarizadas con este servicio y les gusta utilizarlo. Cabe notar, no obstante, que las jóvenes de Nuevo Pedregal de este grupo de edad están algo menos familiarizadas con Internet que las de Andaray y son más temerosas. Esto tiene que ver con su menor interacción con otros actores, por el escaso interés que suscita la localidad para la comunidad de desarrollo. Nuevo Pedregal, en este sentido, sufre la maldición de ser una localidad pobre situada en un departamento próspero. Su situación periférica hace que quede al margen de las dinámicas territoriales, pero sin llegar a calificar para formar parte de muchos programas sociales, que se focalizan a partir de criterios agregados a nivel provincial o distrital. Por el contrario Andaray, al estar situado en la sierra sur, la zona más pobre del país, sí es objeto de atención de los esfuerzos públicos o de instituciones privadas. Aunque en menor medida que otros distritos rurales de Cusco o Ayacucho, tanto los hombres como las mujeres están acostumbrados a participar en actividades de ONG y de programas sociales, lo que limita su timidez e incrementa su competencia práctica, a nivel objetivo (habilidades) y subjetivo (conciencia de derechos).

Otro punto a considerar son las normas de género que aún permanecen vigentes y que condicionan una apropiación diferenciada de las nuevas TIC. A diferencia del celular, para usar Internet las mujeres deben movilizarse a las cabinas, que hasta hoy son el espacio más importante-y en algunos casos el único-para acceder a esta tecnología. Este desplazamiento implica costos en tiempo y dinero, además de suponer una interacción social, que no siempre está bien vista. El control, a veces se ejerce de manera directa y a veces de manera solapada, recubierto de discursos de "riesgo y protección". En el caso de la mujeres rurales jóvenes, se trata de un control que es tanto de género (por sus parejas) como generacional (por sus padres y madres).

Las jóvenes afirman que van mucho menos a las cabinas, en comparación de sus hermanos o amigos. Esto ocurre principalmente porque las cabinas se encuentran fuera de sus localidades y las mujeres tienen menos permisos que sus pares masculinos para movilizarse. Tanto en Nuevo Pedregal como en Andaray, la movilidad para llegar a las localidades donde se encuentran las cabinas es sumamente escasa, por lo que hombres y mujeres deben ir caminando. En el caso de Andaray las jóvenes deben caminar cuarenta minutos para llegar a la cabina más cercana y en el de Nuevo Pedregal, alrededor de diez minutos. Este desplazamiento también se dificulta por la mayor carga laboral que tienen las jóvenes en el hogar. Ellas no solo asisten a la escuela, y hacen sus deberes escolares, sino que se espera que también ayuden en las labores domésticas, bastante más que los hombres, incluso durante los fines de semana. De esta manera, las mujeres 
tienen menos tiempo de ocio que los hombres, tiempo que podrían emplear en las cabinas para usar Internet (Peña y Uribe 2013, para un análisis de estos temas en el marco latinoamericano).

Estas prácticas se complementan con discursos que las refuerzan, a partir de estereotipos de género. Las jóvenes participantes en los grupos focales piensan que los hombres saben "moverse" mejor por Internet, tienen mayor conocimiento y han desarrollado más habilidades que ellas. Por lo tanto, la gama de opciones de qué hacer con Internet es mayor para los hombres. Estas opiniones no pasan tanto por tener diferentes habilidades e intereses, sino por la familiaridad que les atribuyen con herramientas y espacios pensados "más para ellos que para ellas".

Este discurso sobre diferencias de género también se refiere propiamente al uso de Internet. Según las jóvenes, a diferencia de los hombres, ellas no chatean mucho, pues prefieren conversar con sus amigas en el colegio o en sus tiempos libres. Las jóvenes cuentan que casi todos sus compañeros hombres van a las cabinas de Internet a ver videos de música, jugar y chatear "con amigas". Por su lado, ellas escuchan música, cuelgan fotos en sus páginas de las redes sociales, a la vez que miran las páginas de sus pares y hacen sus deberes escolares. Se trataría, por lo tanto, de un uso más pasivo de la tecnología. No obstante, más allá de estos discursos, existen también grietas, ya que en las mismas entrevistas algunas chicas señalan que revisan su correo electrónico periódicamente, que, al igual que los números de celular, los hombres jóvenes piden a las mujeres para realizar un primer acercamiento y mostrar interés por una chica.

\section{Brecha generacional: Internet comienza en la escuela}

Un segundo dato importante derivado del trabajo de campo cualitativo es la existencia de una fuerte brecha generacional, que se percibe especialmente en el uso de Internet. Esto se debe, sobre todo, al momento de contacto con esta tecnología, casi siempre ligado a la escuela. Por un lado, el lento ritmo de penetración en las zonas rurales hace que Internet no estuviera disponible cuando las madres y abuelas de las actuales mujeres rurales jóvenes estudiaron. Por otro lado, es cierto también que en los últimos años se ha producido una masiva incorporación de las mujeres rurales a la escuela, que ahora a diferencia de hace apenas dos décadas estudian tanto como sus pares hombres. (Asensio 2012, Agüero y Barreto 2012, Ames 2013).

Las jóvenes entre 14 y 17 años de Nuevo Pedregal cuentan haber accedido por primera vez a Internet en la sala de cómputo de su escuela. Sin embargo, este uso no parece haber sido muy sistemático, ya que solo tenía lugar durante la clase de informática o cuando había algún profesor disponible, lo que no era muy frecuente. Para muchas ese ha sido su primer-y acaso único-contacto con una computadora, y más aún con Inter 
net. Como mencionamos antes, a diez minutos de Nuevo Pedregal, en Pedregal Grande, se encuentra la cabina más cercana. Ahí acuden los y las jóvenes, aunque las jóvenes lo hacen bastante menos. Por el contrario, las mujeres jóvenes de Andaray, sobre todo aquellas entre 14 y 17 años, están más expuestas al uso de la computadora, pues tienen "aulas virtuales" en el colegio. Sin embargo, en el momento en que hicimos el trabajo de campo a comienzos de 2012, la conexión a Internet estaba aún por llegar al distrito. El acceso más cercano estaba en el pueblo vecino de Yanaquihua, a cuarenta minutos a pie, donde hay una cabina que cobra 2,5 soles (algo menos de un dólar) la hora.

En ambos casos las diferencias en edad marcan una diferencia considerable en el uso y actitud con respecto a esta tecnología. Existe una amplia brecha en términos del acceso entre las mujeres rurales de la generación más joven y sus antecesoras, madres y abuelas. En Nuevo Pedregal, ninguna de las mujeres mayores de 17 años dijo haber usado Internet alguna vez, mientras que todas las menores a 17 sí lo habian hecho. Esto se debe, por un lado, a que las computadoras y el acceso a Internet en el colegio han sido implementados hace pocos años y, por otro, porque casi ninguna de las mujeres mayores a 17 años alcanzó los estudios superiores, donde hubieran podido aprender a usar esta tecnología. Esta situación se une a una falta de motivación actual. Las jóvenes de mayor edad que no saben usar Internet demuestran poco interés o no perciben la importancia de aprender a usarlo. En Andaray, en cambio, aunque también existen estas diferencias, la situación es más matizada.

En el caso de la telefonía celular, las diferencias generacionales no se refieren tanto al acceso como al uso de la tecnología. Mientras mayores son las mujeres rurales, menos usos hacen de los aparatos, limitándose a las funciones básicas, como recibir llamadas. Estos quiebres se ven reforzados por los discursos imperantes, que destacan el carácter ambiguo de estas nuevas tecnologías, como una oportunidad, pero también como un peligro potencial (ociosidad, pornografía, redes de captación de menores, etc.). Aunque estos discursos no son tan fuertes en las zonas visitadas, en comparación con los ámbitos urbanos, igualmente forman parte de un contexto general que influye en las entrevistadas. El primer acercamiento a Internet, suele estar marcado por el temor, incluso entre las mujeres más jóvenes. Se trata de un sentimiento en el que se conjugan la ambivalencia de los discursos sociales sobre las nuevas tecnologías con su propia percepción de falta de competencia práctica, que se traduce en el temor de malograr los aparatos. Como cuenta Mónica, de 16 años, de Nuevo Pedregal:

Yo no lo tocaba, porque [...] ninguna lo tocaba. Que me daba miedo, no lo vaya a malograr [...] [El profesor] nos enseñaba, nos decía, después nos animaba, pero no lo haciamos. Nosotros lo apagábamos, él lo prendia. Después ya el profesor nos fue enseñando.

Más allá de estas diferencias generacionales en acceso y uso, las TIC constituyen, también, un espacio de relación e interacción entre las generaciones diferentes gene- 
raciones de mujeres rurales. Cuando le preguntamos a las jóvenes de mayor edad y a las mujeres de más de 35 años para qué sirve Internet, al margen de si lo usan o no, la mayor parte de las veces, la respuesta fue "para comunicarse". Los usos relacionados con adquirir conocimientos, servicios, u otros relacionados con actividades económicas son menos utilizados. Esto son usos que corresponden a una suerte de segundo momento de uso, una vez que ya están familiarizadas con la tecnología.

La necesidad o el deseo de comunicación constituyen la puerta de entrada y un primer interés para utilizar Internet. Sin embargo, en las dos localidades su uso como medio de comunicación aún no reemplaza al celular, que sigue siendo la herramienta más accesible y preferida para comunicarse. Esto es importante porque la comunicación entre padres e hijas a través del celular no es solo una manera de permanecer en contacto, sino también una forma de control. Las madres consideran que gracias al celular pueden Ilamar a sus hijas en cualquier momento, para saber dónde están. Otras incluso afirman que sus hijas solo utilizan el celular en su presencia, de manera que ellas siempre saben con quiénes y sobre qué hablan.

\section{Otro tipo de brecha: la convivencia}

Como mencionamos, a partir de las historias de vida recogidas durante el trabajo de campo hemos identificado un momento crítico en la vida de las mujeres rurales jóvenes, entre los 16 y 22 años de edad, al que denominamos "punto de quiebre" (Agüero y Barreto 2012). Es en esta etapa cuando muchas de ellas optan por comenzar a convivir y formar un hogar. Se trata de un evento traumático, en la medida en que la convivencia no es, en la mayoría de los casos, una iniciativa ni una decisión de las mujeres, sino sobre todo de sus parejas. Muchas veces son ellas quienes se separan de sus familias y se mudan a casa de su familia política. Con ello ven restringido tanto su espacio vital como su privacidad, al tiempo que pasan a ser consideradas mujeres adultas, con nuevas responsabilidades y mayor control social para la interacción con sus pares.

Es en esta etapa crítica entre la juventud y la vida adulta cuando más se muestra el impacto ambivalente de las nuevas tecnologías en la autonomía de las mujeres rurales. En ambas localidades, encontramos que una de las razones más importantes para que los padres compren un celular a sus hijas cuando aún son solteras es para mantenerse comunicados cuando ellas se desplazan a trabajar o a estudiar fuera de la localidad. El celular es valorado mucho por los padres como herramienta de comunicación con sus hijas, a quienes consideran que deben proteger más que a sus hijos. También las hijas valoran esta comunicación con sus familias, cuando están lejos. Muchas jóvenes recibieron su primer celular como regalo de sus padres, padrinos o familiares cercanos, mientras que otras se los compraron con 
sus primeros sueldos, principalmente para cumplir esta función de comunicación, con sus familias, con sus amigas y también-aunque muy pocas lo admiten - con sus enamorados. En esto las mujeres rurales peruanas se muestran muy similares a las de otros paises en desarrollo (Archambault 2009, 2011 y 2013).

El celular, por lo tanto, puede ser visto como una herramienta de control intergeneracional. Sin embargo, al mismo tiempo es también una herramienta de trasgresión. A pesar de las reglas de sus padres, las mujeres rurales jóvenes encuentran en el uso del celular un espacio de privacidad, de mayor libertad y, en última instancia, de transgresión. Esta función se vuelve aún más importante para ellas si tomamos en cuenta que la mayoría de casas rurales solo tiene uno o dos espacios separados, que se comparten entre todos los miembros del hogar.

Este juego complejo entre control y trasgresión se da aun con más fuerza en las mujeres emparejadas. Algunas de las jóvenes mayores de 17 años que participaron en los grupos focales en Andaray recibieron sus primeros celulares como regalo de sus parejas, sobre todo aquellas que eran convivientes. Por esta razón, uno de los mayores usos que le dan a sus celulares es conversar con sus esposos, que salen desde muy temprano a trabajar a la chacra o a la mina.

Frente a este uso del celular como herramienta de control, en otros casos encontramos, sin embargo, que el mismo hecho de comprarse un celular puede ser un gesto de transgresión, una suerte de acto para reafirmación, para establecer cierto nivel de autonomía en la relación de pareja. Un ejemplo de ello es lo que cuenta Gloria de 34 años, también de Andaray:

Así como ahora, trabajando, hice un esfuerzo, así como cuando pago cualquier cosita y dije... o sea, lo que me gusta a mi es que mi esposo no me compre [...] mis cosas, sino comprarme yo. [...] Porque a veces se amarga y discutimos, como usted sabe en los hogares hay eso, y me dice "para qué compraste celular, para que te estés comunicando", entonces ¿qué hice? yo me lo compré. Yo me lo compro mis cosas.

Más allá de estos extremos, en la mayoría de los casos esta doble dimensión de la tecnología se da de manera simultánea. Otro factor a considerar es la etapa de la vida en que las mujeres rurales se encuentran. El tipo de uso del celular depende en gran medida de si son solteras o conviven con una pareja estable.

Las mujeres convivientes o casadas tienen mayores restricciones para andar por las calles, salir con sus amigas, y deben afrontar una mayor carga laboral. Esto influye, sobre todo, en el uso de Internet que en esta etapa casi desaparece, incluso en el caso de las mujeres que lo utilizaban con anterioridad. Con la nueva carga de responsabilidades, caminar hasta otra localidad para acceder a las cabinas es aún más difícil que cuando eran solteras. 


\section{Conclusiones}

En los últimos años las TIC han logrado expandirse considerablemente por las zonas rurales, generando nuevas dinámicas en la vida cotidiana de sus pobladores. Las TIC no solo han tomado cada vez más importancia como herramientas de comunicación, sino que son también un recurso para incrementar la competencia práctica de sus usuarios y para crear nuevas relaciones sociales. Sin embargo, el potencial de las TIC se ve limitado por diferentes factores ligados al contexto y a la experiencia vital de cada usuario, su edad, género, lugar de residencia, etc. Por esta razón, el aporte de las TIC al desarrollo debe ser entendido desde su relación con cada grupo de usuarios, en un escenario sociocultural específico.

Este estudio ha mostrado que, a pesar de las limitaciones que enfrentan las jóvenes rurales para apropiarse de las TIC, su uso ha permitido la creación de nuevos espacios de interacción y privacidad, muy valorada tanto por las mujeres solteras como por aquellas que tienen pareja. El teléfono celular se ha convertido para las mujeres en un dispositivo personal, donde guardan información que desean mantener en el ámbito privado. A través del chat en Internet, algunas jóvenes también sienten mayor confianza para interactuar con hombres, sin que otras personas lo sepan. Se trata de usos trasgresores de la tecnología, que deben ser contextualizados en un momento de cambio importante en los estilos de vida de las mujeres rurales peruanas, tanto a nivel individual (las entrevistadas están en la etapa de tránsito de la juventud a la vida adulta), como a nivel colectivo (en un periodo de importantes transformaciones para las mujeres rurales del Perú).

El mayor acceso a la telefonía celular e Internet, especialmente los grupos de menor edad, han dado paso a nuevas dinámicas sociales que tienen consecuencias no solo en la vida de cada joven, sino en sus familias y comunidades. En las localidades analizadas la introducción de telefonía móvil e Internet es bastante reciente y su impacto todavía es limitado. Sin embargo, de manera incipiente las TIC están abriendo la posibilidad de incrementar de manera significativa el capital social de las mujeres rurales jóvenes y de generar nuevos espacios de individuación, que a su vez resultan en mayores márgenes de autonomía. De esta manera, se puede hablar de una reconfiguración de brechas de desigualdad que afectan a las jóvenes rurales (Asensio 2012).

Uno de los principales usos de la telefonía celular es mantener la comunicación del grupo familiar de referencia. En ese sentido, encontramos, por un lado, que son las mujeres jóvenes quienes asumen el rol de mantener vínculos emocionales con familiares y amistades, no solo con los que viven en su comunidad, sino con aquellos que se encuentran lejos. Mantienen conversaciones frecuentes por celular con sus padres cuando se encuentran trabajando o estudiando lejos. Cuando empiezan a convivir con sus parejas y dejan el hogar, las jóvenes se sienten menos solas y más seguras, pues sienten mayor apoyo de sus padres y de sus amigas, al poder hablar con ellos por el celular. Al mismo tiempo, el celular le da a los padres más seguridad respecto a dónde están sus hijas. Esta nueva 
relación puede incluso traducirse en mayores permisos para movilizarse e interactuar con sus pares, en distintos espacios de la localidad o incluso fuera de ella.

El uso del celular y de Internet permite también que se configuren nuevas relaciones, no solo amicales sino también amorosas. En contextos rurales, donde los espacios domésticos suelen estar saturados, las nuevas TIC proporcionan un espacio de privacidad fuera de la vigilancia de los padres y de otros miembros de la comunidad. Con el uso de estas tecnologías se vuelven más frecuentes las relaciones a larga distancia, pues las mujeres pueden conversar con sus parejas, amigos o amigas con mayor frecuencia, lo que permite consolidar un capital social incipiente. Podemos hablar en este sentido de un "campo invisible" (Archambault 2009), para manejar relaciones de manera discreta, aumentando los márgenes de libertad y autonomía, y desafiando las relaciones de poder y control.

Las nuevas generaciones de mujeres rurales jóvenes interactúan con mayor frecuencia con los hombres y construyen vínculos más estrechos con sus compañeras del colegio y con otras amigas de la comunidad. Es posible, en este sentido, que su vida futura sea menos solitaria que la de sus madres y abuelas. Un valor añadido de la extensión de Internet para las jóvenes rurales es el hecho de que les permite salir de casa. Acudir a las cabinas públicas, a pesar de las restricciones que hemos señalado, es un evento social que permite interacciones diferentes a las habituales. Las interacciones cara a cara, se suman con otras nuevas, a través de llamadas telefónicas, mensajes de texto, redes sociales y chats. Igual que los hombres, las mujeres usan estas herramientas como entretenimiento, aunque recubierto con un discurso que pasa por la escuela y los deberes escolares, que permiten afirmar la "necesidad" de usar de Internet. Es con ese pretexto que sus padres les dan permiso y el dinero para acceder a las cabinas públicas (sin que ello signifique que no sea verdad que hagan tareas utilizando la web).

En Andaray y Nuevo Pedregal existe una valoración positiva del celular e Internet. No existe aún un discurso que perciba estas tecnologías como elementos "corruptores" o riesgosos. Las mujeres rurales aún no se plantean la posibilidad de que sus hijos o sus pares incurran en conductas indebidas incitadas por su relación con las TIC. Este es un riesgo que ha sido señalado en otros casos, pero que aún no parece haberse concretado (Archambault 2013). Tanto en Andaray como en Nuevo Pedregal, las comunicaciones a través del celular son principalmente con amigos y familiares mientras que las comunicaciones por Internet son también bastantes restringidas. Quienes tienen correo afirman que cuidan mucho a quién se lo dan y las que chatean lo hacen con personas conocidas. En este sentido no existe un discurso que valore positivamente el comportamiento alocado o la imagen "chonguera", similar al existente en las jóvenes urbanas del mismo rango de edad (León 2013).

Las mujeres rurales jóvenes encuentran en las nuevas TIC espacios de privacidad, crecimiento, autoaprendizaje y autorrealización. Sin embargo, esto no quiere decir que las brechas y limitaciones hayan desaparecido. Persisten, como hemos visto, brechas de desigualdad en torno al acceso y uso de las tecnologías. Junto con la extensión y consoli- 
dación del capital social, a la creación de nuevos ámbitos de sociabilidad y a la extensión de los márgenes de individuación, las TIC también han propiciado nuevas prácticas y discursos de control sobre las mujeres rurales jóvenes. Este control es tanto generacional (por sus padres y madres) como de género (por sus parejas hombres)'1.

También está pendiente el reto de la profundización del uso de la tecnología. En las dos zonas analizadas encontramos casos en que los usuarios han aprendido a usar el celular o las computadoras por sí mismos. Sin ninguna o muy poca capacitación, han logrado manipular estas herramientas correctamente y aprender a usarlas para su propio beneficio: para conseguir trabajos eventuales, para comunicarse con sus familiares y amigos o simplemente para entretenerse. Sin embargo, se trata de casos excepcionales en un contexto que todavía es poco propicio. La oferta de servicios es limitada, sobre todo en el caso de Internet. $\mathrm{Ni}$ en las escuelas ni en las cabinas, existe un acompañamiento acorde a las necesidades de los usuarios, y pocas veces se incluyen contenidos relevantes para su realidad de mujeres rurales jóvenes. La mayoría de jóvenes rurales no ve Internet como un medio de desarrollo o aprendizaje, sino más bien solo como una forma de comunicación y entretenimiento.

El reto de las TIC, pasa por ampliar la oferta de servicios y hacerse más relevante para la demanda específica rural. Es necesario que existan iniciativas estatales, que vayan más allá de la expansión del acceso y se concentren en los procesos de uso y apropiación de las tecnologías. Es importante que se generen espacios en los que las jóvenes rurales puedan aprender a utilizar estas herramientas, y que al mismo tiempo consideren las características, los procesos y las dinámicas de los espacios rurales. Estas iniciativas también deben incluir temas de género, y cumplir con las necesidades de atención y asistencia de las mujeres rurales. Como plantean Phillippi y Peña (2012), deben propiciar no solo el desarrollo de las habilidades digitales sino también estar orientadas a sus expectativas, y a acompañarlas en la realización de sus propios sueños, yendo más allá de sus quehaceres diarios en la escuela y en el hogar.

\section{Referencias}

Agüero, A., y M. Barreto. (2012). El nuevo perfil de las mujeres rurales jóvenes en el Perú. Lima: Instituto de Estudios Peruanos, Documentos del Programa Nuevas Trenzas, 2. Ames, P. (2013). ¿Construyendo nuevas identidades? Género y educación en los proyectos de vida de las jóvenes rurales del Perú, Lima: Instituto de Estudios Peruanos, Documentos del Programa Nuevas Trenzas, XXX.

Anderson, J. (2001). Tendiendo puentes. Calidad de atención desde la perspectiva de muje

1 Un tema pendiente de analizar es si la extensión de las nuevas TIC está generando en las zonas rurales formas de control por pares, similares a las descritas por León (2013) en los ámbitos urbanos. 
res rurales y de los proveedores de los servicios de salud. Lima: Manuela Ramos, 2001. Archambault, J.S. (2009). "Being cool or being good: researching mobile phones in Mozambique". Anthropology Matters Journal, 11(2): 1-9.

Archambault, J.S. (2011). "Breaking up "because of the phone" and the transformative potential of information in Southern Mozambique". New Media Society, 13(3): 444-456.

Archambault, J.S. (2013). "Cruising through uncertainty: Cell phones and the politics of display and disguise in Inhambane, Mozambique". American Ethnologist, 40(1): 88-10.

Aronés, M., R. Barrantes y L. León. (2011). "Todos tienen celular": uso, apropiación e impacto de la telefonía móvil en el área de influencia de dos ferias en Puno, Perú. Lima: Instituto de Estudios Peruanos, Documento de trabajo 161.

Asensio, R. H. (2012). Nuevas (y viejas) historias sobre las mujeres rurales jóvenes de América Latina. Resultados preliminares del programa Nuevas Trenzas. Lima: Instituto de Estudios Peruanos, Documentos del Programa Nuevas Trenzas, 1.

Banco Mundial (2012). Informe sobre el desarrollo mundial 2012: Igualdad de género y desarrollo. Washington DC: Banco Mundial.

Barrantes, R., R. Cuenca y J. Morel. (2012). Las posibilidades del desarrollo inclusivo: dos historias regionales. Lima: Instituto de Estudios Peruanos.

Barreto, M., y A. García. (2012). "Brechas que se cierran, brechas que se abren: Las mujeres rurales jóvenes en el desarrollo territorial rural". Ponencia presentada en el Encuentro Territorios Rurales en Movimiento, Quito, 6 de junio de 2012.

Barreto, M., y A. García. (2013). “¿Nuevas estrategias de sociabilidad de mujeres rurales jóvenes?: el impacto de las TIC en dos comunidades rurales de Piura y Arequipa" en A. Paz, M. P. Montoya y R. H. Asensio, Escalando innovaciones rurales. Lima: Instituto de Estudios Peruanos: International Development Research Centre: Fondo Internacional para el Desarrollo Agrícola, pp. 239-257.

Benitez Larghi, et al. (2012). "La apropiación de computadoras y acceso a Internet por parte de jóvenes de sectores populares urbanos en la Argentina" en F. J. Proenza, en Tecnología y cambio social: El impacto del acceso público a las computadoras e internet en Argentina, Chile y Perú. Lima, IDRC-CRDI; IEP. Pp. 70-104

Bossio, J. F., et al. (2004). Desarrollo rural y tecnologías de información y comunicación. Lima: Sociedad Alemana de Cooperación Técnica: Intermediate Technology Development Group: Ministerio de Agricultura, Dirección General de Información Agraria.

Buskens, I., y Webb, A. (2009). African Women \& ICTs: Investigating Technology, Gender and Empowerment. London \& New York: Zed Books

Burrell, J. (2010). "User Agency in the Middle Range: Rumors and the Reinvention of the Internet in Accra, Ghana". Science, Technology \& Human Values, 8(2): 1-21

Bustamante, R., Z. Burneo y M. Alvarado. (2009). Usos efectivos y necesidades de información para el desarrollo de estrategias apropiadas para proyectos TIC en el área rural. Lima: Centro Peruano de Estudios Sociales. 
Calcina, L. A. (2013). "TICs para la Amazonía: ¿conectando el desarrollo?" en A. Paz, M.P. Montoya y R. H. Asensio, Escalando innovaciones rurales. Lima: Instituto de Estudios Peruanos: International Development Research Centre: Fondo Internacional para el Desarrollo Agrícola, pp. 223-238.

Donner, J. (2008). "Research Approaches to Mobile Use in the Developing World: A Review of the Literature". The Information Society, 24 (3): 140-159.

Escobal, J., y C. Ponce. (2012). Polarización y segregación en la distribución del ingreso en el Perú: trayectorias desiguales. Lima: Grupo de Análisis para el Desarrollo, Documento de Investigación, 62.

Hahn, P., y L. Kibora. (2008). "The domestication of the mobile phone: oral society and new ICT in Burkina Faso". Journal of Modern African Studies, 46 (1): 87-109.

Hopkins, R. et al. (2013). "Las cabinas de Internet como un instrumento de desarrollo e inclusión de los pueblos alto andinos del sur del Perú" en A. Paz, M.P. Montoya y R. H. Asensio, Escalando innovaciones rurales. Lima: Instituto de Estudios Peruanos: International Development Research Centre: Fondo Internacional para el Desarrollo Agrícola, pp. 203-222.

León, D. (2013). "Feminidades en conflicto y conflictos entre mujeres: Construcción de feminidades, formas de transgresión y violencia en adolescentes de dos colegios públicos de Lima". Ponencia presentada en el Instituto de Estudios Peruanos, Lima, 31 de enero de 2013.

León Kanashiro, L. (2010). "Adolescentes y web 2.0", en Barindelli, F. y C. G. Gregorio, Datos personales y libertad de expresión en las redes sociales digitales. Memorándum de Montevideo. Buenos Aires: Ad-Hoc.

Peña, X., y C. Uribe. (2013). Economía del cuidado. Visibilización y valoración del trabajo femenino no remunerado en América Latina. Lima: Instituto de Estudios Peruanos, Documentos del Programa Nuevas Trenzas, 16Phillippi, A. y P. Peña. (2012). "Impacto del acceso público en dos telecentros: apropiación social de las TIC por parte de mujeres chilenas" en Proenza, Francisco J., Tecnología y cambio social: El impacto del acceso público a las computadoras e internet en Argentina, Chile y Perú. Lima, IDRC-CRDI; IEP. Pp. 70-104

Quiroz, M.T. (2010) "Jóvenes y relaciones interactivas". Ponencia presentada en el Congreso Educomunicación, Medellín, 30 de setiembre al 2 de octubre de 2010.

Ramírez, R. (2007). "Appreciating the Contribution of Broadband ICT With Rural and Remote Communities: Stepping Stones. Toward an Alternative Paradigm" En: The Information Society, 23: 85.94 